\begin{tabular}{l|l}
-1.3 & -0.5 \\
-1.4 & +0.2 \\
-1.6 & +0.9 \\
-1.5 & +1.2 \\
-1.2 & +1.2
\end{tabular}

Vergl. die Curve in Fig. III.

Wie man sieht, ist das vorstehende Beispiel nicht geeignet, die gegebene Theorie zu illustriren, obgleich auch hier die Verbesserung des Anschlusses unverkennbar ist. Man hat es wohl diesem Umstande zuzuschreiben, dass dasselbe so vielfach in Lehrbüchern aufgeführt wird, um den Anschluss der Erfahrung an das Gauss'sche Fehlergesetz darzuthun, wozu allerdings die beiden vorhergehenden Beispiele weniger passend wären. Uebrigens sei hier nochmals daran erinnert, dass der Ausdruck von $W(v)$ keineswegs die Grundlage einer allgemeinen Fehlertheorie sein soll.

IV) Das vierte Beispiel endlich ergiebt:

$$
\begin{aligned}
& x=+0.3103 \pm 0.0660 \\
& y=+0.1348 \pm 0.0391
\end{aligned}
$$

Die Einsetzung in die Formel für $W(v)$ ergab folgende Unterschiede von der Gauss'schen Theorie:

$$
\begin{array}{r|r}
+1.1 & -0.5 \\
+0.4 & -0.3 \\
-0.3 & -0.1 \\
-0.6 & 0.0
\end{array}
$$

Mit Hülfe dieser Zahlen ist die Curve in Fig. IV construirt, die einen ziemlich guten Anschluss giebt. Fassen wir die Zahlen in ähnlicher Weise zusammen, wie es im ersten Beispiel geschehen war, so erhalten wir:

Fehlergrenzen

in Einheiten des w. F.

$$
\begin{array}{r}
E-G \\
+1.5 \\
-0.8 \\
-10 \\
+0.4
\end{array}
$$$$
2.0 \cdot 3.0
$$

während die Abweichungen unserer Theorie von der Gauss' schen folgende werden:

$$
\begin{array}{l|l}
+1.5 & -0.8 \\
-0.9 & -0.1
\end{array}
$$

Die. Quadratsumme der Abweichungen wird hier von 4.0 auf 0.3 reducirt. Vergl. IV $V^{a}$.

Die gegebenen Entwickelungen zeigen, dass, wenn auch durchaus nicht in allen, so doch in gewissen Fällen unser Ausdruck für die Fehlerwahrscheinlichkeit der wahren Vertheilung ziemlich gut entspricht. Man sieht, dass in diesen Fällen das zu häufige Auftreten grosser Fehler meist ein nur scheinbares ist, so dass an eine Verwerfung von Beobachtungen hier nicht zu denken ist. Was das letzte Beispiel betrifft, so ist allerdings das Ueberwiegen grösserer Fehler nicht beseitigt, allein eine bessere Uebereinstimmung von einer Reihe von nur 15 Beobachtungen zu erwarten, ist völlig unberechtigt. Durch eine Aenderung der Constanten $x$ und $y$ könnte man leicht diesen störenden $U m$ stand beseitigen, wenn man dafür etwas von der Genauigkeit des Gesammtanschlusses aufgiebt. Dass das Gesetz, nach welchem wir $h$ variiren liessen, ein hypothetisches ist, darf freilich nicht vergessen werden, und aus diesem Grunde wird man selten eine derartige Uebereinstimmung zwischen Theorie und Erfahrung finden, wie in unserem zweiten Beispiel.

Gesetzt aber auch, dass sich derartige Gesetzmässig: keiten, wie wir sie untersucht haben, nicht entdecken lassen, so wird doch die Verwerfung von Beobachtungen immer eine sehr bedenkliche Sache bleiben, da ja eine regellos eingetretene Störung den Beobachtungsfehler ebenso gut verkleinern wie vergrössern kann. Es wird aber offenbar Niemand einfallen, Beobachtungen mit kleinen Beobachtungsfehlern zu verwerfen, die, ohne dass man es weiss, demselben Genauigkeitsgrade angehören können, wie sehr

\begin{tabular}{|c|c|c|c|c|c|}
\hline I 88 I & M. Z. Kasan & \multicolumn{2}{|c|}{$\alpha$ app. } & $\delta$ app. & $\log p .4$ \\
\hline Juni 24 & I I $25^{\mathrm{m}} 40$ & $5^{\mathrm{h}} 3^{8}$ & m.5.94 & $+48^{\circ} 49^{\prime} 25^{\prime \prime} 5$ & 0.932 \\
\hline 25 & I I 2552 & $5 \quad 42$ & I 5.2 I & +52493 & .923 \\
\hline 29 & I I 32 I 2 & 6 & 22 & $-65 \quad 27 \quad 19.1$ & 2.879 \\
\hline Juli & $\begin{array}{lll}\text { I I } & 39 & 39\end{array}$ & 619 & 43.84 & $+695^{8} 3^{8.2}$ & 0.856 \\
\hline 5 & I $2 \quad 6 \quad$ I 4 & 7 & 9.40 & +76 I9 55.6 & 0.817 \\
\hline 8 & I $2 \quad 38 \quad 19$ & 746 & 9.68 & +791824.0 & 0.796 \\
\hline
\end{tabular}
fehlerhafte. Etwas Anderes wäre es allerdings, wenn in

\begin{tabular}{|c|c|c|c|c|c|c|c|c|}
\hline \multicolumn{2}{|c|}{$188 I$} & \multirow{2}{*}{$\frac{\text { M. Z. Kasan }}{{ }^{1} 2^{\mathrm{h}} 5^{\mathrm{m}} 18^{\mathrm{s}}}$} & \multicolumn{3}{|c|}{$\alpha$ app. } & \multicolumn{2}{|c|}{$\delta$ app. } & \multirow{2}{*}{$\frac{\log p .4}{0.790}$} \\
\hline uli & 9 & & & $3^{\text {in }}$ & 7.20 & $+80^{\circ}$ & & \\
\hline & IO & I $3 \quad 5 \quad 14$ & 8 & 21 & 2.5 & +803 & 752.8 & 0.786 \\
\hline & I I & I3 $19 \quad 57$ & 8 & 39 & 44. & +81 & 724.5 & 0.782 \\
\hline & I 2 & I3 $35 \quad 9$ & 8 & $5^{8}$ & & +8 . 3 & 57.8 & 0.779 \\
\hline & 13 & I3 5034 & 9 & I 8 & & $+8 I 4$ & $57 \cdot 9$ & 0.776 \\
\hline & 14 & $1455^{\circ}$ & 9 & 37 & $34.5^{2}$ & $+8 z$ & $2 \quad 3.2$ & 0.774 \\
\hline
\end{tabular}
einer sonst gut übereinstimmenden Beobachtungsreihe einige Beobachtungen vorkämen, deren Fehler von einer ganz anderen Ordnung sind als die ibrigen.

Berlin 1887 Juni.

\title{
Beobachtungen des Cometen 1881 III
}

in der unteren Culmination am Repsold'schen Meridiankreise der Universitäts-Sternwarte $z$ u Kasan.

Juli 8 und 14 Comet in Wolken; Juli 9 Bild unruhig; Juli $\mathbf{2} 2 \delta$ app. ist vergrössert um $z^{\prime}$. Die Oerter der Vergleichsterne sind dem Fundamental. Catalog der AG. von Auwers entnommen. Den $\log p . \Delta$ habe ich mit der Parallaxe $=8.85$ berechnet.

Kasan I 887 Mai i r.

Dr. P. Poretzki, Observator der Sternwarte. 